\title{
Exponential Growth of Solutions for Nonlinear Coupled Viscoelastic Wave Equations
}

\author{
Erhan Pişkin $^{1 *}$ and Şeyhmus Altındağ ${ }^{1}$ \\ ${ }^{1}$ Department of Mathematics, Dicle University, 21280 Diyarbakır, Turkey \\ * Corresponding author
}

\section{Article Info}

Keywords: Exponential growth, Nonlinear damping term, Viscoelastic wave equations.

2010 AMS: 35B44, 35L52.

Received: 17 November 2018

Accepted: 9 April 2019

Available online: 28 June 2019

\section{Abstract}

In this work, we consider an initial-boundary value problem related to the nonlinear coupled viscoelastic equations

$$
\left\{\begin{array}{c}
\left|u_{t}\right|^{j} u_{t t}-\Delta u_{t t}-\operatorname{div}\left(|\nabla u|^{\alpha-2} \nabla u\right)-\Delta u+\int_{0}^{t} g(t-s) \Delta u d s+\left|u_{t}\right|^{m-1} u_{t}=f_{1}(u, v) \\
\left|v_{t}\right|^{j} v_{t t}-\Delta v_{t t}-\operatorname{div}\left(|\nabla v|^{\beta-2} \nabla v\right)-\Delta v+\int_{0}^{t} h(t-s) \Delta v d s+\left|v_{t}\right|^{r-1} v_{t}=f_{2}(u, v)
\end{array}\right.
$$

We will show the exponential growth of solutions with positive initial energy.

\section{Introduction}

In this work we consider the following coupled system of viscoelastic wave equations:

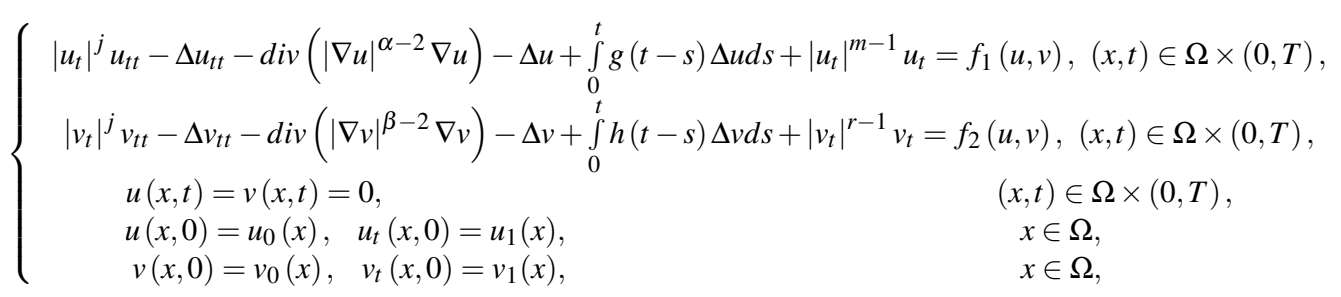

where $\Omega$ is a bounded domain in $R^{n}(n=1,2,3)$ with smooth boundary $\partial \Omega$, the constants $j>0, \alpha \geq 2, \beta \geq 2, m \geq 1, r \geq 1$. Here, $f_{1}(u, v)$ and $f_{2}(u, v)$ are nonlinear functions defined as

$$
\left\{\begin{array}{c}
f_{1}(u, v)=a|u+v|^{2(p+1)}(u+v)+b|u|^{p} u|v|^{p+2} \\
f_{2}(u, v)=a|u+v|^{2(p+1)}(u+v)+b|v|^{p} v|u|^{p+2}
\end{array}\right.
$$

in which the constants $a>0, b>0$, and $p$ satisfies

$$
\left\{\begin{array}{c}
p>-1, \quad n=1,2 \\
-1<p \leq 1, \quad n=3
\end{array}\right.
$$

Let

$$
f_{1}(u, v)=\frac{\partial F(u, v)}{\partial u} \text { and } f_{2}(u, v)=\frac{\partial F(u, v)}{\partial v}
$$


where

$$
F(u, v)=\frac{1}{2(p+2)}\left[a|u+v|^{2(p+2)}(u+v)+2 b|u v|^{p+2}\right] .
$$

There are two positive constants $c_{0}, c_{1}$ such that

$$
c_{0}\left(|u|^{2(r+2)}+|v|^{2(r+2)}\right) \leq 2(r+2) F(u, v) \leq c_{1}\left(|u|^{2(r+2)}+|v|^{2(r+2)}\right) .
$$

As a special case, for $\alpha=\beta=2$, the system (1.1) becomes the following system

$$
\left\{\begin{array}{l}
\left|u_{t}\right|^{j} u_{t t}-\Delta u_{t t}-\Delta u+\int_{0}^{t} g(t-s) \Delta u d s+\left|u_{t}\right|^{m-1} u_{t}=f_{1}(u, v), \\
\left|v_{t}\right|^{j} v_{t t}-\Delta v_{t t}-\Delta v+\int_{0}^{t} h(t-s) \Delta v d s+\left|v_{t}\right|^{r-1} v_{t}=f_{2}(u, v) .
\end{array}\right.
$$

Liu [1] proved decay of the solutions for system (1.4) under some appropriate functions $f_{1}$ and $f_{2}$. Later, Said-Houari [2] studied exponential growth of the solutions for system (1.4). When $j=0$ and without the $\Delta u_{t t}, \Delta v_{t t}$ terms, the system (1.4) has been investigated by some authors and results concerning local and global existence, blow up, decay of the solutions were obtained [3, 4, 5, 6, 7, 8]. Hao et al. [9] considered global nonexistence of the solution of (1.1), with negative initial energy.

Motivated by the above papers, in this work we prove the exponential growth of solutions for the problem (1.1), with positive initial energy. This work is organized as follows: In section 2, we present some lemmas and notations needed later of this paper. In section 3 , exponential growth of the solution is proved.

\section{Preliminaries}

In this part, we give some assumptions and lemmas which will be used throughout this paper. Let $\|\cdot\|$ and $\|\cdot\|_{p}$ denote the usual $L^{2}(\Omega)$ norm and $L^{p}(\Omega)$ norm, respectively.

Now, we make the following assumptions on the $C^{1}$-nonnegative and nonincreasing relaxation functions $g$ and $h$ :

$$
1-\int_{0}^{\infty} g(s) d s=l>0, \quad 1-\int_{0}^{\infty} h(s) d s=k>0
$$

and $\forall s \geq 0$

$$
g^{\prime}(s) \leq 0, h^{\prime}(s) \leq 0
$$

Let us define

$$
\begin{aligned}
I(t) & =I(u, v)=\left(1-\int_{0}^{t} g(s) d s\right)\|\nabla u\|^{2}+\left(1-\int_{0}^{t} h(s) d s\right)\|\nabla v\|^{2} \\
& -2(p+2) \int_{\Omega} F(u, v) d x+(g \circ \nabla u+h \circ \nabla v)+\frac{1}{\alpha}\|\nabla u\|_{\alpha}^{\alpha}+\frac{1}{\beta}\|\nabla v\|_{\beta}^{\beta}, \\
J(t) & =J(u, v)=\frac{1}{2}\left(1-\int_{0}^{t} g(s) d s\right)\|\nabla u\|^{2}+\frac{1}{2}\left(1-\int_{0}^{t} h(s) d s\right)\|\nabla v\|^{2} \\
& -\int_{\Omega} F(u, v) d x+\frac{1}{2}(g \circ \nabla u+h \circ \nabla v)+\frac{1}{\alpha}\|\nabla u\|_{\alpha}^{\alpha}+\frac{1}{\beta}\|\nabla v\|_{\beta}^{\beta}
\end{aligned}
$$

and

$$
\begin{aligned}
E(t) & =\frac{1}{j+2}\left(\left\|u_{t}\right\|_{j+2}^{j+2}+\left\|v_{t}\right\|_{j+2}^{j+2}\right)+\frac{1}{2}\left(\left\|\nabla u_{t}\right\|^{2}+\left\|\nabla v_{t}\right\|^{2}\right) \\
& +\frac{1}{2}\left(1-\int_{0}^{t} g(s) d s\right)\|\nabla u\|^{2}+\frac{1}{2}\left(1-\int_{0}^{t} h(s) d s\right)\|\nabla v\|^{2} \\
& -\int_{\Omega} F(u, v) d x+\frac{1}{2}(g \circ \nabla u+h \circ \nabla v)+\frac{1}{\alpha}\|\nabla u\|_{\alpha}^{\alpha}+\frac{1}{\beta}\|\nabla v\|_{\beta}^{\beta}
\end{aligned}
$$

where

$$
(\phi \circ \psi)(t)=\int_{0}^{t} \phi(t-\tau) \int_{\Omega}|\psi(t)-\psi(\tau)|^{2} d x d \tau .
$$


Lemma 2.1. $E(t)$ is a nonincreasing function for $t \geq 0$ and

$$
\begin{aligned}
E^{\prime}(t) & =-\left(\left\|u_{t}\right\|_{m+1}^{m+1}+\left\|v_{t}\right\|_{m+1}^{m+1}\right)+\frac{1}{2}\left(g^{\prime} \circ \nabla u+h^{\prime} \circ \nabla v\right) \\
& -\frac{1}{2}\left(g(t)\|\nabla u\|^{2}+h(t)\|\nabla v\|^{2}\right) \\
& \leq 0 .
\end{aligned}
$$

Proof. Multiplying the first and second equation of (1.1) by $u_{t}$ and $v_{t}$, respectively, integrating over $\Omega \times[0, t]$, then adding them together and integrating by parts, we obtain (2.6).

\section{Exponential growth of solutions}

In this part, we are going to consider the exponential growth of the solution for the problem (1.1). Firstly, we give following two lemmas.

Lemma 3.1. [10, 11]. Suppose that (1.3) holds. Let $(u, v)$ for $\eta>0$

$$
\begin{aligned}
\|u+v\|_{2(p+2)}^{2(p+2)}+2\|u v\|_{p+2}^{p+2} & \leq \eta\left[\frac{1}{\alpha}\|\nabla u\|_{\alpha}^{\alpha}+\frac{1}{\beta}\|\nabla v\|_{\beta}^{\beta}\right. \\
& \left.+I_{1}\|\nabla u\|^{2}+I_{2}\|\nabla v\|^{2}\right]^{p+2},
\end{aligned}
$$

where

$$
\begin{aligned}
& I_{1}=\int_{\Omega_{1}}\left|u_{t}\right|\left(|u|^{2 p+3}+|v|^{2 p+3}+|u|^{p+1}|v|^{p+2}\right) d x \\
& I_{2}=\int_{\Omega_{2}}\left|v_{t}\right|\left(|u|^{2 p+3}+|v|^{2 p+3}+|u|^{p+2}|v|^{p+1}\right) d x
\end{aligned}
$$

and

$$
\begin{aligned}
& \Omega_{1}=\{(x, t):|u(x, t)| \leq 1,|v(x, t)| \leq 1\}, \\
& \Omega_{2}=\{(x, t):|u(x, t)| \leq 1,|v(x, t)| \geq 1\} .
\end{aligned}
$$

Lemma 3.2. $[10,11]$. Suppose that (1.3) holds. Let $(u, v)$ be the solution of problem (1.1). Assume further that $E(0)<E_{1}$ and

$$
\left[\frac{1}{\alpha}\left\|\nabla u_{0}\right\|_{\alpha}^{\alpha}+\frac{1}{\beta}\left\|\nabla v_{0}\right\|_{\beta}^{\beta}+I(0)\right]^{\frac{1}{2}}>\alpha_{1} .
$$

Then, there exists a constant $\alpha_{2}>\alpha_{1}$ such that

$$
\begin{aligned}
& {\left[\frac{1}{\alpha}\|\nabla u\|_{\alpha}^{\alpha}+\frac{1}{\beta}\|\nabla v\|_{\beta}^{\beta}+I(t)\right]^{\frac{1}{2}}>\alpha_{2},} \\
& \left(\|u+v\|_{2(p+2)}^{2(p+2)}+\|u v\|_{p+2}^{p+2}\right)^{\frac{1}{2(p+2)}}>B \alpha_{2},
\end{aligned}
$$

for all $t \in(0, T)$, where

$$
B=\eta^{\frac{1}{2(p+2)}}, \alpha_{1}=B^{-\frac{p+2}{p+1}}, E_{1}=\left(\frac{1}{2}-\frac{1}{2(p+2)}\right) \alpha_{1}^{2} .
$$

Theorem 3.3. Suppose that (1.3) holds. Assume further that

$$
\begin{aligned}
& \max \{j+2, m+1, r+1\}<2(p+2), \\
& E(0)<E_{1}
\end{aligned}
$$

and (2.1), (2.2) hold. There exist constant $\gamma$ such that

$$
\max \{\alpha, \beta\}<\gamma<2(p+2)
$$

and

$$
\min \{l, k\}>\frac{1 /(2 \gamma)}{(\gamma / 2)-1+1 /(2 \gamma)} .
$$

Then, any solution of (1.1) grows exponentially. 
Proof. We define the functional

$$
H(t)=E_{1}-E(t)
$$

From (2.1), (2.5) and Lemma 3.2, we have

$$
\begin{aligned}
0 & <H(0) \leq H(t) \\
& \leq E_{1}-E(t) \\
& =E_{1}-\frac{1}{j+2}\left(\left\|u_{t}\right\|_{j+2}^{j+2}+\left\|v_{t}\right\|_{j+2}^{j+2}\right)-\frac{1}{2}\left(\left\|\nabla u_{t}\right\|^{2}+\left\|\nabla v_{t}\right\|^{2}\right) \\
& -\frac{1}{2}\left(1-\int_{0}^{t} g(s) d s\right)\|\nabla u\|^{2}-\frac{1}{2}\left(1-\int_{0}^{t} h(s) d s\right)\|\nabla v\|^{2} \\
& +\int_{\Omega} F(u, v) d x-\frac{1}{2}(g \circ \nabla u+h \circ \nabla v)-\frac{1}{\alpha}\|\nabla u\|_{\alpha}^{\alpha}-\frac{1}{\beta}\|\nabla v\|_{\beta}^{\beta} \\
& <E_{1}-\frac{1}{2} \alpha_{2}^{2}+\frac{1}{2(p+2)}\left(\|u\|_{2(p+2)}^{2(p+2)}+\|v\|_{2(p+2)}^{2(p+2)}\right) \\
& <\frac{C_{1}}{2(p+2)}\left(\|u\|_{2(p+2)}^{2(p+2)}+\|v\|_{2(p+2)}^{2(p+2)}\right) .
\end{aligned}
$$

Let us define the functional

$$
L(t)=H(t)+\frac{\varepsilon}{j+1} \int_{\Omega}\left(\left|u_{t}\right|^{j} u_{t} u+\left|v_{t}\right|^{j} v_{t} v\right) d x-\varepsilon \int_{\Omega}\left(\Delta u u_{t}+\Delta v v_{t}\right) d x
$$

where $\varepsilon$ is a small positive constants to be determined later.

By differentiating with respect to $t$ and using (3.3) and (1.1), we have

$$
\begin{aligned}
L^{\prime}(t) & =H^{\prime}(t)+\varepsilon \int_{\Omega}\left[\left(\left|u_{t}\right|^{j} u_{t t} u+\left|v_{t}\right|^{j} v_{t t} v\right)+\frac{1}{j+1}\left(\left|u_{t}\right|^{j+2}+\left|v_{t}\right|^{j+2}\right)\right] d x \\
& +\varepsilon\left(\left\|\nabla u_{t}\right\|^{2}+\left\|\nabla v_{t}\right\|^{2}\right)-\varepsilon \int_{\Omega}\left(u \Delta u_{t t}+v \Delta v_{t t}\right) d x \\
& =H^{\prime}(t)+\frac{\varepsilon}{j+1}\left(\left\|u_{t}\right\|_{j+2}^{j+2}+\left\|v_{t}\right\|_{j+2}^{j+2}\right)-\varepsilon \int_{\Omega}\left(u\left|u_{t}\right|^{m-1} u_{t}+v\left|v_{t}\right|^{r-1} v_{t}\right) d x \\
& +\varepsilon\left(\left\|\nabla u_{t}\right\|^{2}+\left\|\nabla v_{t}\right\|^{2}\right)-\varepsilon\left(\|\nabla u\|^{2}+\|\nabla v\|^{2}\right)-\varepsilon\left(\|\nabla u\|_{\alpha}^{\alpha}+\|\nabla v\|_{\beta}^{\beta}\right) \\
& +2 \varepsilon(p+2) \int_{\Omega} F(u, v) d x+\varepsilon\left(\int_{0}^{t} g(s) d s\right)\|\nabla u\|^{2}+\varepsilon\left(\int_{0}^{t} h(s) d s\right)\|\nabla v\|^{2} \\
& +\varepsilon \int_{0}^{t} g(t-s) \int_{\Omega} \nabla u[\nabla u(s)-\nabla u(t)] d x d s \\
& +\varepsilon \int_{0}^{t} h(t-s) \int_{\Omega} \nabla v[\nabla v(s)-\nabla v(t)] d x d s .
\end{aligned}
$$

Using Cauchy-Schwarz and Young's inequalities, we get

$$
\begin{aligned}
\int_{0}^{t} g(t-s) \int_{\Omega} \nabla u[\nabla u(s)-\nabla u(t)] d x d s & \leq \int_{0}^{t} g(t-s)\left(\int_{\Omega}|\nabla u(t)|^{2} d x\right)^{\frac{1}{2}}\left(\int_{\Omega}|\nabla u(s)-\nabla u(t)|^{2} d x\right)^{\frac{1}{2}} d s \\
& \leq \int_{0}^{t} g(t-s)\|\nabla u(t)\|\|\nabla u(s)-\nabla u(t)\| d s \\
& \leq \int_{0}^{t} g(t-s)\left(\lambda\|\nabla u(s)-\nabla u(t)\|^{2}+\frac{1}{4 \lambda}\|\nabla u(t)\|^{2}\right) d s \\
& \leq \lambda \int_{0}^{t} g(t-s)\|\nabla u(s)-\nabla u(t)\|^{2} d s+\frac{1}{4 \lambda} \int_{0}^{t} g(t-s)\|\nabla u(t)\|^{2} d s \\
& \leq \lambda(g \circ \nabla u)+\frac{1}{4 \lambda}\left(\int_{0}^{t} g(s) d s\right)\|\nabla u(t)\|^{2} .
\end{aligned}
$$


Similarly, we obtain

$$
\int_{0}^{t} h(t-s) \int_{\Omega} \nabla v[\nabla v(s)-\nabla v(t)] d x d s \leq \lambda(h \circ \nabla v)+\frac{1}{4 \lambda}\left(\int_{0}^{t} h(s) d s\right)\|\nabla v(t)\|^{2} .
$$

Inserting (3.5) and (3.6) into (3.4), we have

$$
\begin{aligned}
L^{\prime}(t) & \geq H^{\prime}(t)+\frac{\varepsilon}{j+1}\left(\left\|u_{t}\right\|_{j+2}^{j+2}+\left\|v_{t}\right\|_{j+2}^{j+2}\right)-\varepsilon \int_{\Omega}\left(u\left|u_{t}\right|^{m-1} u_{t}+v\left|v_{t}\right|^{r-1} v_{t}\right) d x \\
& +\varepsilon\left(\left\|\nabla u_{t}\right\|^{2}+\left\|\nabla v_{t}\right\|^{2}\right)-\varepsilon\left(\|\nabla u\|^{2}+\|\nabla v\|^{2}\right)-\varepsilon\left(\|\nabla u\|_{\alpha}^{\alpha}+\|\nabla v\|_{\beta}^{\beta}\right) \\
& +2 \varepsilon(p+2) \int_{\Omega} F(u, v) d x+\varepsilon\left(\int_{\Omega} g(s) d s\right)\|\nabla u\|^{2}+\varepsilon\left(\int_{\Omega} h(s) d s\right)\|\nabla v\|^{2} \\
& +\varepsilon \lambda(g \circ \nabla u+h \circ \nabla v)+\frac{\varepsilon}{4 \lambda}\left[\left(\int_{0}^{t} g(s) d s\right)\|\nabla u\|^{2}+\left(\int_{0}^{t} h(s) d s\right)\|\nabla v\|^{2}\right] .
\end{aligned}
$$

By the definition of $E(t)$ and (3.1), we obtain

$$
\begin{aligned}
\int_{\Omega} F(u, v) d x & =H(t)-E_{1}+\frac{1}{j+2}\left(\left\|u_{t}\right\|_{j+2}^{j+2}+\left\|v_{t}\right\|_{j+2}^{j+2}\right)+\frac{1}{2}\left(\left\|\nabla u_{t}\right\|^{2}+\left\|\nabla v_{t}\right\|^{2}\right) \\
& +\frac{1}{2}\left(1-\int_{0}^{t} g(s) d s\right)\|\nabla u\|^{2}+\frac{1}{2}\left(1-\int_{0}^{t} h(s) d s\right)\|\nabla v\|^{2} \\
& +\frac{1}{2}(g \circ \nabla u+h \circ \nabla v)+\frac{1}{\alpha}\|\nabla u\|_{\alpha}^{\alpha}+\frac{1}{\beta}\|\nabla v\|_{\beta}^{\beta} .
\end{aligned}
$$

Substituting (3.8) into (3.7), we get

$$
\begin{aligned}
L^{\prime}(t) & \geq H^{\prime}(t)+\varepsilon\left(\frac{1}{j+1}+\frac{\gamma}{j+2}\right)\left(\left\|u_{t}\right\|_{j+2}^{j+2}+\left\|v_{t}\right\|_{j+2}^{j+2}\right)-\varepsilon \int_{\Omega}\left(u\left|u_{t}\right|^{m-1} u_{t}+v\left|v_{t}\right|^{r-1} v_{t}\right) d x \\
& +\varepsilon\left(1+\frac{\gamma}{2}\right)\left(\left\|\nabla u_{t}\right\|^{2}+\left\|\nabla v_{t}\right\|^{2}\right)+\gamma \varepsilon H(t)-\varepsilon \gamma E_{1}+\varepsilon(2(p+2)-\gamma) \int_{\Omega} F(u, v) d x \\
& +\varepsilon\left[\left(\frac{\gamma}{2}-1\right)-\left(\frac{\gamma}{2}-1+\frac{1}{4 \lambda}\right) \int_{0}^{\infty} g(s) d s\right]\|\nabla u\|^{2} \\
+ & {\left[\left(\frac{\gamma}{2}-1\right)-\left(\frac{\gamma}{2}-1+\frac{1}{4 \lambda}\right) \int_{0}^{\infty} h(s) d s\right]\|\nabla v\|^{2} } \\
+ & {\left[\left(\frac{\gamma}{2}-\lambda\right)(g \circ \nabla u+h \circ \nabla v)+\varepsilon\left(\frac{\gamma}{\alpha}-1\right)\|\nabla u\|_{\alpha}^{\alpha}+\varepsilon\left(\frac{\gamma}{\beta}-1\right)\|\nabla v\|_{\beta}^{\beta} .\right.}
\end{aligned}
$$

By using the Young's inequality, we get

$$
\begin{aligned}
\int_{\Omega}\left|u_{t}\right|^{m-1} u_{t} u d x & \leq \frac{\delta_{1}^{m+1}}{m+1}\|u\|_{m+1}^{m+1}+\frac{m \delta_{1}^{-\frac{m+1}{m}}}{m+1}\left\|u_{t}\right\|_{m+1}^{m+1} \\
& \leq \frac{\delta_{1}}{m+1}\|u\|_{m+1}^{m+1}+\frac{m \delta_{1}^{-\frac{m+1}{m}}}{m+1} H^{\prime}(t)
\end{aligned}
$$

and

$$
\begin{aligned}
\int_{\Omega}\left|v_{t}\right|^{r-1} v_{t} v d x & \leq \frac{\delta_{2}^{r+1}}{r+1}\|v\|_{r+1}^{r+1}+\frac{r \delta_{2}^{-\frac{r+1}{r}}}{r+1}\left\|v_{t}\right\|_{r+1}^{r+1} \\
& \leq \frac{\delta_{2}^{r+1}}{r+1}\|v\|_{r+1}^{r+1}+\frac{r \delta_{2}^{-\frac{r+1}{r}}}{r+1} H^{\prime}(t) .
\end{aligned}
$$

Since $L^{2(p+2)}(\Omega) \hookrightarrow L^{m+1}(\Omega)$ and $L^{2(p+2)}(\Omega) \hookrightarrow L^{r+1}(\Omega)$, we have

$$
\left(\|u\|_{2(p+2)}^{2(p+2)}+\|v\|_{2(p+2)}^{2(p+2)}\right)^{m}\|u\|_{m+1}^{m+1} \leq C_{2}\left(\|u\|_{2(p+2)}^{2(p+2)}+\|v\|_{2(p+2)}^{2(p+2)}\right)^{m+\frac{m+1}{2(p+2)}}
$$

and

$$
\left(\|u\|_{2(p+2)}^{2(p+2)}+\|v\|_{2(p+2)}^{2(p+2)}\right)^{r}\|v\|_{r+1}^{r+1} \leq C_{3}\left(\|u\|_{2(p+2)}^{2(p+2)}+\|v\|_{2(p+2)}^{2(p+2)}\right)^{r+\frac{r+1}{2(p+2)}} .
$$


We use the following algebraic inequality

$$
z^{v} \leq z+1 \leq\left(1+\frac{1}{a}\right)(z+a), \quad \forall z \geq 0,0<v \leq 1,
$$

we obtain, for $t \geq 0$,

$$
\begin{aligned}
\left(\|u\|_{2(p+2)}^{2(p+2)}+\|v\|_{2(p+2)}^{2(p+2)}\right)^{m+\frac{m+1}{2(p+2)}} & \leq d\left(\|u\|_{2(p+2)}^{2(p+2)}+\|v\|_{2(p+2)}^{2(p+2)}+H(0)\right) \\
& \leq d\left(\|u\|_{2(p+2)}^{2(p+2)}+\|v\|_{2(p+2)}^{2(p+2)}+H(t)\right)
\end{aligned}
$$

and

$$
\begin{aligned}
\left(\|u\|_{2(p+2)}^{2(p+2)}+\|v\|_{2(p+2)}^{2(p+2)}\right)^{r+\frac{r+1}{2(p+2)}} & \leq d\left(\|u\|_{2(p+2)}^{2(p+2)}+\|v\|_{2(p+2)}^{2(p+2)}+H(0)\right) \\
& \leq d\left(\|u\|_{2(p+2)}^{2(p+2)}+\|v\|_{2(p+2)}^{2(p+2)}+H(t)\right)
\end{aligned}
$$

for $d=1+\frac{1}{H(0)}$.

By (3.9)-(3.13),(3.15) and (3.16), we have

$$
\begin{aligned}
L^{\prime}(t) & \geq\left(1+\frac{m \delta_{1}^{-\frac{m+1}{m}}}{m+1}+\frac{r \delta_{2}^{-\frac{r+1}{r}}}{r+1}\right) H^{\prime}(t)+\varepsilon\left(\frac{1}{j+1}+\frac{\gamma}{j+2}\right)\left(\left\|u_{t}\right\|_{j+2}^{j+2}+\left\|v_{t}\right\|_{j+2}^{j+2}\right) \\
& -\left(\frac{\delta_{1}^{m+1} c_{2} d}{m+1}+\frac{\delta_{2}^{r+1} c_{3} d}{r+1}\right)\left(\|u\|_{2(p+2)}^{2(p+2)}+\|v\|_{2(p+2)}^{2(p+2)}\right) \\
& +\varepsilon\left(\gamma-\left(\frac{\delta_{1}^{m+1} c_{2} d}{m+1}+\frac{\delta_{2}^{r+1} c_{3} d}{r+1}\right)\right) H(t) \\
& +\varepsilon\left(1+\frac{\gamma}{2}\right)\left(\|\nabla u\|^{2}+\|\nabla v\|^{2}\right)+\varepsilon(2(p+2)-\gamma(p+2)) \int_{\Omega} F(u, v) d x \\
& +\varepsilon\left[\left(\frac{\gamma}{2}-1\right)-\left(\frac{\gamma}{2}-1+\frac{1}{4 \lambda}\right) \int_{0}^{\infty} g(s) d s\right]\|\nabla u\|^{2} \\
& +\varepsilon\left(\frac{\gamma}{2}-\lambda\right)(g \circ \nabla u+h \circ \nabla v) \\
& +\varepsilon\left[\left(\frac{\gamma}{2}-1\right)-\left(\frac{\gamma}{2}-1+\frac{1}{4 \lambda}\right) \int_{0}^{\infty} h(s) d s\right]\|\nabla v\|^{2} \\
& +\varepsilon\left(\frac{\gamma}{\alpha}-1\right)\|\nabla u\|_{\alpha}^{\alpha}+\varepsilon\left(\frac{\gamma}{\beta}-1\right)\|\nabla v\|_{\beta}^{\beta} .
\end{aligned}
$$

By use (3.2) and since

$$
\min \left\{\frac{\gamma}{\alpha}-1, \frac{\gamma}{\beta}-1\right\}>0
$$

and

$$
1+\frac{\gamma}{2}>0
$$

we obtain

$$
\begin{aligned}
L^{\prime}(t) & \geq M H^{\prime}(t)+\varepsilon\left(\frac{1}{j+1}+\frac{\gamma}{j+2}\right)\left(\left\|u_{t}\right\|_{j+2}^{j+2}+\left\|v_{t}\right\|_{j+2}^{j+2}\right)+\varepsilon\left(\gamma-K_{1}\right) H(t) \\
& +\varepsilon K_{2}\left(\|\nabla u\|_{\alpha}^{\alpha}+\|\nabla v\|_{\beta}^{\beta}\right)+\varepsilon K_{3}\left(\|\nabla u\|^{2}+\|\nabla v\|^{2}\right) \\
& +\varepsilon\left(\frac{\gamma}{2}-\lambda\right)(g \circ \nabla u+h \circ \nabla v)+\varepsilon\left(1+\frac{\gamma}{2}\right)\left(\left\|\nabla u_{t}\right\|^{2}+\left\|\nabla v_{t}\right\|^{2}\right) \\
& +\varepsilon\left(\frac{(2(p+2)-\gamma(p+2)) C_{1}}{2(p+2)}-K_{1}\right)\left(\|u\|_{2(p+2)}^{2(p+2)}+\|v\|_{2(p+2)}^{2(p+2)}\right)
\end{aligned}
$$

where

$$
\begin{aligned}
M & =1+\frac{m \delta_{1}^{-\frac{m+1}{m}}}{m+1}+\frac{r \delta_{2}^{-\frac{r+1}{r}}}{r+1}, \\
K_{1} & =\frac{\delta_{1}^{m+1} c_{2} d}{m+1}+\frac{\delta_{2}^{r+1} c_{3} d}{r+1}, \\
K_{2} & =\min \left\{\frac{\gamma}{\alpha}-1, \frac{\gamma}{\beta}-1\right\}
\end{aligned}
$$


and

$$
K_{3}=\left(\frac{\gamma}{2}-1\right)-\left(\frac{\gamma}{2}-1+\frac{1}{4 \lambda}\right) \max \left(\int_{0}^{\infty} g(s) d s, \int_{0}^{\infty} h(s) d s\right) .
$$

Choose $\delta_{1}, \delta_{2}$ appropriate such that

$$
b_{1}=\gamma-K_{1}>0, b_{2}=\frac{(2(p+2)-\gamma(p+2)) C_{1}}{2(p+2)}-K_{1}>0 \text { and } M>0 .
$$

Then, we can find positive constants $b_{1}$ and $b_{2}$ such that

$$
\begin{aligned}
L^{\prime}(t) & \geq M H^{\prime}(t)+\varepsilon\left(\frac{1}{j+1}+\frac{\gamma}{j+2}\right)\left(\left\|u_{t}\right\|_{j+2}^{j+2}+\left\|v_{t}\right\|_{j+2}^{j+2}\right) \\
& +\varepsilon K_{2}\left(\|\nabla u\|_{\alpha}^{\alpha}+\|\nabla v\|_{\beta}^{\beta}\right)+\varepsilon\left(1+\frac{\gamma}{2}\right)\left(\left\|\nabla u_{t}\right\|^{2}+\left\|\nabla v_{t}\right\|^{2}\right) \\
& +\varepsilon b_{1} H(t)+\varepsilon b_{2}\left(\|u\|_{2(p+2)}^{2(p+2)}+\|v\|_{2(p+2)}^{2(p+2)}\right) \geq 0 .
\end{aligned}
$$

Because of $H^{\prime}(t) \geq 0$, there exists constants $t>0$ such that

$$
\begin{aligned}
L^{\prime}(t) & \geq \tilde{K}\left(H(t)+\left\|u_{t}\right\|_{j+2}^{j+2}+\left\|v_{t}\right\|_{j+2}^{j+2}+\|\nabla u\|_{\alpha}^{\alpha}+\|\nabla v\|_{\beta}^{\beta}\right. \\
& \left.+\left\|\nabla u_{t}\right\|^{2}+\left\|\nabla v_{t}\right\|^{2}+\|u\|_{2(p+2)}^{2(p+2)}+\|v\|_{2(p+2)}^{2(p+2)}\right) \geq 0
\end{aligned}
$$

where $\tilde{K}=\min \left\{\varepsilon b_{1}, \varepsilon\left(\frac{1}{j+1}+\frac{\gamma}{j+2}\right), \varepsilon K_{2}, \varepsilon\left(1+\frac{\gamma}{2}\right), \varepsilon b_{2}\right\}$.

On the other hand, we can choose $\varepsilon$ smaller so that

$$
L(0)=H(0)+\varepsilon \int_{\Omega}\left(u_{0} u_{1}+v_{0} v_{1}\right) d x>0 .
$$

Furthermore, we have

$$
L(t) \geq L(0), t \geq 0 .
$$

Next we estimate $L(t)$. Using Young's inequality, we obtain

$$
\left.\left|\int_{\Omega}\right| u_{t}\right|^{j+1} u d x \mid \leq \frac{\mu_{1}^{j+2}}{j+2}\|u\|_{j+2}^{j+2}+\frac{(j+1) \mu_{1}^{-\frac{j+2}{j+1}}}{j+2}\left\|u_{t}\right\|_{j+2}^{j+2}, \forall \mu_{1}>0 .
$$

Next, using the embedding $L^{2(p+2)}(\Omega) \hookrightarrow L^{j+2}(\Omega)$, the estimate (3.20) becomes

$$
\begin{aligned}
\left.\left|\int_{\Omega}\right| u_{t}\right|^{j+1} u d x \mid & \leq C\left(\|u\|_{2(p+2)}^{j+2}+\left\|u_{t}\right\|_{j+2}^{j+2}\right) \\
& \leq C\left(\left(\|u\|_{2(p+2)}^{2(p+2)}\right)^{\frac{j+2}{2(p+2)}}+\left\|u_{t}\right\|_{j+2}^{j+2}\right) .
\end{aligned}
$$

Since $2(p+2)>j+2$ and $H(t)>H(0)$, use the inequality (3.14), we have

$$
\begin{aligned}
\left.\left|\int_{\Omega}\right| u_{t}\right|^{j+1} u d x \mid & \leq C\left[\left(1+\frac{1}{H(0)}\right)\left(\|u\|_{2(p+2)}^{2(p+2)}+H(0)\right)+\left\|u_{t}\right\|_{j+2}^{j+2}\right] \\
& \leq C\left[\left(1+\frac{1}{H(0)}\right)\left(\|u\|_{2(p+2)}^{2(p+2)}+H(t)\right)+\left\|u_{t}\right\|_{j+2}^{j+2}\right] .
\end{aligned}
$$

Similarly, we have

$$
\left.\left|\int_{\Omega}\right| v_{t}\right|^{j+1} v d x \mid \leq C\left[\left(1+\frac{1}{H(0)}\right)\left(\|v\|_{2(p+2)}^{2(p+2)}+H(t)\right)+\left\|v_{t}\right\|_{j+2}^{j+2}\right] .
$$

By Green identity and Hölder's inequality, we get

$$
\begin{aligned}
-\int_{\Omega} u_{t} \Delta u d x & =\int_{\Omega} \nabla u \nabla u_{t} d x \\
& \leq\left(\int_{\Omega}(\nabla u)^{2} d x\right)^{\frac{1}{2}}\left(\int_{\Omega}\left(\nabla u_{t}\right)^{2} d x\right)^{\frac{1}{2}} \\
& =\|\nabla u\|\left\|\nabla u_{t}\right\|
\end{aligned}
$$


similarly

$$
-\int_{\Omega} v_{t} \Delta v d x \leq\|\nabla v\|\left\|\nabla v_{t}\right\| .
$$

Next, using the embedding $L^{\alpha}(\Omega) \hookrightarrow L^{2}(\Omega)$ and $L^{\beta}(\Omega) \hookrightarrow L^{2}(\Omega)$ the estimate (3.23) and (3.24) becomes

$$
\left\{\begin{aligned}
\|\nabla u\|\left\|\nabla u_{t}\right\| & \leq C\|\nabla u\|_{\alpha}\left\|\nabla u_{t}\right\| \\
\|\nabla v\|\left\|\nabla v_{t}\right\| & \leq C\|\nabla v\|_{\beta}\left\|\nabla v_{t}\right\|
\end{aligned}\right.
$$

By Young's inequality (3.25), we get

$$
\begin{gathered}
\|\nabla u\|_{\alpha}\left\|\nabla u_{t}\right\| \leq \frac{1}{2}\left(\|\nabla u\|_{\alpha}^{2}+\left\|\nabla u_{t}\right\|^{2}\right), \\
\|\nabla v\|_{\beta}\left\|\nabla v_{t}\right\| \leq \frac{1}{2}\left(\|\nabla v\|_{\alpha}^{2}+\left\|\nabla v_{t}\right\|^{2}\right) .
\end{gathered}
$$

Since $\alpha \geq 2, \beta \geq 2$ and $H(t)>H(0)$, the inequality (3.14) yields

$$
\begin{aligned}
\|\nabla u\|_{\alpha}^{2} & =\left(\|\nabla u\|_{\alpha}^{\alpha}\right)^{\frac{2}{\alpha}} \\
& \leq\left(1+\frac{1}{H(0)}\right)\left(\|\nabla u\|_{\alpha}^{\alpha}+H(0)\right) \\
& \leq\left(1+\frac{1}{H(0)}\right)\left(\|\nabla u\|_{\alpha}^{\alpha}+H(t)\right)
\end{aligned}
$$

and

$$
\begin{aligned}
\|\nabla v\|_{\beta}^{2} & =\left(\|\nabla v\|_{\beta}^{\beta}\right)^{\frac{2}{\beta}} \\
& \leq\left(1+\frac{1}{H(0)}\right)\left(\|\nabla v\|_{\beta}^{\beta}+H(0)\right) \\
& \leq\left(1+\frac{1}{H(0)}\right)\left(\|\nabla v\|_{\beta}^{\beta}+H(t)\right) .
\end{aligned}
$$

Combining (3.20)-(3.28), we have

$$
\begin{aligned}
& \left|\frac{\varepsilon}{j+1} \int_{\Omega}\left(\left|u_{t}\right|^{j} u_{t} u+\left|v_{t}\right|^{j} v_{t} v\right) d x-\varepsilon \int_{\Omega}\left(\Delta u u_{t}+\Delta v v_{t}\right) d x\right| \\
& \leq \mu\left(H(t)+\left\|u_{t}\right\|_{j+2}^{j+2}+\left\|v_{t}\right\|_{j+2}^{j+2}+\|\nabla u\|_{\alpha}^{\alpha}+\|\nabla v\|_{\beta}^{\beta}+\left\|\nabla u_{t}\right\|^{2}+\left\|\nabla v_{t}\right\|^{2}\right. \\
& \left.+\|u\|_{2(p+2)}^{2(p+2)}+\|v\|_{2(p+2)}^{2(p+2)}\right) .
\end{aligned}
$$

Thus, we obtain

$$
\begin{aligned}
L(t) & \leq C^{*}\left(H(t)+\left\|u_{t}\right\|_{j+2}^{j+2}+\left\|v_{t}\right\|_{j+2}^{j+2}+\|\nabla u\|_{\alpha}^{\alpha}+\|\nabla v\|_{\beta}^{\beta}+\left\|\nabla u_{t}\right\|^{2}+\left\|\nabla v_{t}\right\|^{2}\right. \\
& \left.+\|u\|_{2(p+2)}^{2(p+2)}+\|v\|_{2(p+2)}^{2(p+2)}\right) .
\end{aligned}
$$

A combination of (3.17) and (3.29) yields

$$
L(t) \leq C^{*} L^{\prime}(t) \text { for all } t \geq 0
$$

where $C^{*}$ is a some positive constants. Integrating the differential inequality (3.30) between 0 and $t$ gives the following estimate for $L(t)$,

$$
L(t) \geq L(0) e^{t / C^{*}}
$$

This completes the proof.

\section{Conclusion}

In this paper, we obtained a exponential growth of solutions for a nonlinear coupled viscoelastic wave equations with nonlinear damping terms. This improves and extends many results in the literature such as (Houari [2], Pişkin [5]).

\section{Acknowledgement}

The authors are grateful to DUBAP (ZGEF.17.009) for research funds. 


\section{References}

[1] W. Liu, Uniform decay of solutions for a quasilinear system of viscoelastic equations, Nonlinear Anal., 71 (2009) $2257-2267$.

[2] B.S. Houari, Exponential growth of positive initial-energy solutions of a system of nonlinear viscoelastic wave equations with damping and source terms, Z. Angew. Math. Phys., 62 (2011) 115-133.

[3] X. Han , M. Wang, Global existence and blow-up solutions for a system of nonlinear viscoelastic wave equations with damping and source, Nonlinear Anal., 71 (2009) 5427-5450.

[4] S.A. Messaoudi , B.S. Houari, Global nonexistence of positive initial-energy solutions of a system of nonlinear viscoelastic wave equations with damping and source terms, J. Math. Anal. Appl., 365 (2010) 277-287.

[5] E. Pişkin, A lower bound for the blow up time of a system of viscoelastic wave equations with nonlinear damping and source terms, J. Nonlinear Funct. Anal., 2017 (2017) 1-9.

[6] E. Pişkin, Global nonexistence of solutions for a system of viscoelastic wave equations with weak damping terms, Malaya Journal of Matematik, 3(2) (2015) 168-174.

[7] B.S. Houari, S.A. Messaoudi, A. Guesmia, General decay of solutions of a nonlinear system of viscoelastic wavw equations, Nonlinear Differ. Equ. Appl., 18 (2011) 659-684.

[8] Y. Zhao, Q. Wang, Blow-up of arbitrarily positive initial energy solutions for a viscoelastic wave system with nonlinear damping and source terms, Boundary Value Problems, 35 (2018) 1-13.

[9] J. Hao, S. Niu, H. Men, Global nonexistence of solutions for nonlinear coupled viscoelastic wave equations with damping and source terms, Boundary Value Problems, 250 (2014) 1-11.

[10] L. Fei, G. Hongjun, Global nonexistence of positive initial energy solutions for coupled nonlinear wave equations with damping and source terms, Abst. Appl. Anal., 2011 (2011) 1-14.

[11] J. Hao, L. Cai, Global existence and blow up of solutions for nonlinear coupled wave equations with viscoelastic terms, Math. Meth. Appl. Sci., 39 (2016) 1977-1989. 